\title{
Hydrogen-rich medium suppresses the generation of reactive oxygen species, elevates the Bcl-2/Bax ratio and inhibits advanced glycation end product-induced apoptosis
}

\author{
HONG JIANG ${ }^{1}$, PAN YU ${ }^{2}$, DE-HUI QIAN ${ }^{1}$, ZHE-XUE QIN ${ }^{1}$, XUE-JUN SUN ${ }^{3}$, JIE YU $^{1}$ and LAN HUANG ${ }^{1}$ \\ ${ }^{1}$ Institute of Cardiovascular Diseases of PLA, Xinqiao Hospital, Third Military Medical University, Chongqing 400037; \\ ${ }^{2}$ Department of Burns and Plastic Surgery, Jinling Hospital, School of Medicine, Nanjing University, \\ Nanjing, Jiangsu 210002; ${ }^{3}$ Department of Diving Medicine, Faculty of Naval Medicine, \\ Second Military Medical University, Shanghai 200433, P.R. China
}

Received December 20, 2012; Accepted February 20, 2013

DOI: 10.3892/ijmm.2013.1334

\begin{abstract}
The purpose of the present study was to determine whether using hydrogen-rich medium (HRM) to increase hydrogen levels in endothelial cells (ECs) protects ECs from apoptosis induced by advanced glycation end products (AGEs). The thoracic aorta was removed from 2-3-year-old SpragueDawley rats, and ECs were isolated and cultured. After culturing ECs in the presence of AGEs and/or with HRM for $24 \mathrm{~h}$, Annexin V/7-AAD and TUNEL staining were carried out to detect apoptosis. Intracellular ROS were detected by fluorescent probe and quantified by flow cytometry. The expression of antioxidative enzymes (superoxide dismutase, glutathione peroxidase) was determined by real-time PCR analysis and enzymatic assay. The relative expression levels of Bcl-2 and Bax were analyzed by western blotting. The addition of AGEs increased the apoptosis of ECs in a concentration-dependent manner and HRM reduced the AGE ( $400 \mu \mathrm{g} / \mathrm{ml})$-induced apoptosis from $21.61 \pm 2.52$ to $11.32 \pm 1.75 \%$. HRM also significantly attenuated the AGE-induced intracellular ROS induction and decrease in the expression of antioxidative enzymes. In conclusion, hydrogen exhibits significant protective effects against AGE-induced EC injury possibly through reducing ROS generation, intracellular antioxidant enzyme system protection and elevation of the Bcl-2/Bax ratio.
\end{abstract}

\section{Introduction}

Cardiovascular complications in diabetes mellitus are one of the leading causes of patient mortality. Vascular endothelial injury and dysfunction are important early pathological mani-

Correspondence to: Dr Lan Huang, Institute of Cardiovascular Diseases of PLA, Xinqiao Hospital, Third Military Medical University, Chongqing 400037, P.R. China

E-mail: drhuanglan@163.com

Key words: advanced glycation end product, apoptosis, endothelial cell, hydrogen, reactive oxygen species festations of the cardiovascular complications of diabetes (1). Increased apoptosis is an early manifestation of endothelial injury, leading to endothelial dysfunction (2).

Advanced glycation end products (AGEs) are the product of non-enzymatic glycosylation on the amino groups of macromolecules such as proteins and nucleic acids (3). In diabetic patients, sustained high blood glucose significantly increases production of AGEs (4). Epidemiological studies show that the presence of AGEs is highly correlated with diabetic cardiovascular complications (4). Previous studies found that AGEs increase endothelial cell (EC) apoptosis and dysfunction (5). In addition, our previous study found that AGEs increase apoptosis and dysfunction of endothelial precursor cells (6). The generation of intracellular reactive oxygen species (ROS) increases in AGE-induced cells, and oxidative stress and ROS production, in turn, contribute to AGE-induced apoptosis of ECs and endothelial precursor cells (6).

Removal of AGEs from the body is challenging. One strategy to reduce AGE-induced endothelial injury is to antagonize AGE-activated oxidative stress (7). Hydrogen $\left(\mathrm{H}_{2}\right)$ is the smallest gas molecule in nature. Studies have shown that $\mathrm{H}_{2}$ has antioxidant activities in living organisms; it can specifically neutralize the most potent oxidative free radicals $(\cdot \mathrm{OH}$ and $\mathrm{ONOO}^{-}$) and it can also attenuate the superoxidant anion level in certain pathophysiological conditions (8). Moreover, it is easy for $\mathrm{H}_{2}$ to pass through membrane structures such as cell membranes and the mitochondrial membrane, where it can neutralize intracellular ROS, thereby maintaining normal mitochondrial function and preventing apoptosis (9). Numerous studies have shown that $\mathrm{H}_{2}$ or hydrogen-containing solution can alleviate ischemia-reperfusion injury (10-12) of the heart (13), brain (14,15), kidney, small intestine, and liver (16); it can also antagonize irradiation-induced cell injury (17). Inhalation of $\mathrm{H}_{2}$ also slows down the growth of atherosclerotic plaque in apoE $\mathrm{E}^{-/-}$mice (18). Our previous studies also found that hydrogen-rich saline prevented neointima formation following carotid balloon injury (19). Oxidative stress plays an important role in ischemia-reperfusion injury, irradiation-induced injury, atherosclerosis and neointima formation (17). The ability of $\mathrm{H}_{2}$ to antagonize these pathological reactions is closely related to 
the anti-ROS effects of $\mathrm{H}_{2}$. However, whether $\mathrm{H}_{2}$ can ameliorate AGE-induced ROS generation and apoptosis of ECs has yet to be elucidated.

The purpose of the present study was to determine whether use of hydrogen-rich medium (HRM) can protect the ECs from AGE-induced apoptosis, by detecting ROS production and antioxidant-related gene expression.

\section{Materials and methods}

Animals. This study used 2-3-year-old Sprague-Dawley rats purchased from the Experimental Animal Center of the Third Military Medical University, Chongqing, China. All protocols conformed to the Guide for the Care and Use of Laboratory Animals published by the US National Institutes of Health (NIH Publication no. 85-23, revised 1996).

Preparation of HRM. HRM was prepared as previously described (18). Briefly, $\mathrm{H}_{2}$ was dissolved in low-glucose Dulbecco's modified Eagle's medium (DMEM-L; Hyclone, Logan, UT, USA) supplemented with $20 \%$ fetal bovine serum (FBS) for $6 \mathrm{~h}$ under high pressure $(0.4 \mathrm{Mpa})$. The saturated HRM was stored at $4^{\circ} \mathrm{C}$ under atmospheric pressure in an aluminum bag with no dead volume. To ensure an $\mathrm{H}_{2}$ concentration of $>0.6 \mathrm{mmol} / \mathrm{l}$, the medium was freshly prepared every week. $\mathrm{H}_{2}$ concentration in the prepared medium was confirmed with gas chromatography as previously described (8).

Primary cell culture. Rat aortic ECs were isolated and cultured as previously described (20). In brief, the thoracic aorta was removed from the Sprague-Dawley rats and placed into a 100-mm culture dish (Corning, Inc., Corning, NY, USA) filled with serum-free DMEM-L (Hyclone) on ice. The adipose tissue and adventitia of the aorta were removed. The aorta was placed intimal side down on a sterile plate containing $0.2 \%$ collagenase type I (Sigma, St. Louis, MO, USA) and incubated at $37^{\circ} \mathrm{C}$ for $30 \mathrm{~min}$. Detached ECs were collected, cultured in DMEM-L supplemented with 20\% FBS (Gibco, USA), $100 \mathrm{U} / \mathrm{ml}$ penicillin, $100 \mu \mathrm{g} / \mathrm{ml}$ streptomycin and $75 \mu \mathrm{g} / \mathrm{ml}$ EC growth supplement (Sigma) and placed in a $50 \mathrm{ml}$ culture flask (Corning, Inc.). ECs at passages 3-5 were used in the experiments. The purity of ECs was evaluated by detection of von Willebrand factor (vWF) expression by fluorescence immunocytochemistry using anti-vWF antibody (Santa Cruz Biotechnology, Inc., Santa Cruz, CA, USA). More than $95 \%$ of ECs in the culture were vWF positive. In the experiments, cells were incubated with various concentrations of AGEs (Jingmei Biotech Co., Ltd, China) for $24 \mathrm{~h}$.

Apoptosis assays. For Annexin $\mathrm{V}$ and 7-aminoactinomycin D (7-AAD) assays, Annexin $\mathrm{V}$ binding and 7-AAD staining were carried out using the Annexin V-APC/7-AAD Apoptosis Detection Kit (Keygen Biotechnology, Nanjing, China). ECs following treatment were trypsinized, washed twice and resuspended in binding buffer to a concentration of $1 \times 10^{6}$ cells $/ \mathrm{ml}$. A volume of $500 \mu \mathrm{l}$ of cells was mixed with $5 \mu \mathrm{l}$ of Annexin V-APC and 7-AAD for $10 \mathrm{~min}$ at room temperature in the dark. After adding binding buffer $(400 \mu \mathrm{l})$, cells were analyzed by flow cytometry (FACSCalibur; BD Biosciences). For terminal uridine deoxynucleotidyl transferase dUTP nick end labelling (TUNEL) staining, cells were plated on coverglasses for $16 \mathrm{~h}$ and fixed and were then incubated with $0.1 \%$ Triton X-100 for 2 min and washed twice with PBS. TUNEL staining was performed as previously described (21).

Detection of ROS. DCF-DA (Sigma) was used as a fluorescent probe to detect intracellular ROS, and flow cytometry was used to determine the fluorescence intensity of cells. The spontaneous fluorescence intensity of the negative control tube without DCF-DA was defined as 1, and the fluorescence intensity values of other groups were the values relative to that of the negative control (detected fluorescence intensity/the fluorescence intensity of the negative control). The treated cells in each group were collected and $5 \mathrm{ml}$ serum-free medium and $10 \mathrm{mM}$ DCF-DA were added into a $50 \mathrm{ml}$ culture flask. The cells were incubated with $5 \% \mathrm{CO}_{2}$ at $37^{\circ} \mathrm{C}$ for $45 \mathrm{~min}$; the cells were then washed with PBS and digested with $0.25 \%$ trypsin. After the digestion was terminated, the cells were washed with PBS twice and were resuspended in $2 \mathrm{ml}$ serum-free medium. In the positive control group, ROS inducer Rosup (50 $\mu \mathrm{g} / \mathrm{ml})$ was added together with DCF-DA, and the cells were incubated at room temperature for $1 \mathrm{~h}$ prior to fluorescent detection. The fluorescent intensity of the sample tubes represents the amount of intracellular ROS.

Detection of antioxidative enzymes. Levels of superoxide dismutase (SOD) and glutathione peroxidase (GSH-PX) expression were analyzed by real-time PCR analysis. Total RNA was purified from cultured ECs with TRIzol (Invitrogen, USA) according to the manufacturer's protocol. Total RNA was reverse-transcribed into cDNA, and the cDNA product was amplified by SYBR-Green I fluorescence realtime PCR. The PCR reaction [containing $12.5 \mu 1$ RNase-free distilled water, $10 \mu 1$ SYBR-Green I master mix (Toyobo Co., Ltd., Osaka, Japan), $0.75 \mu \mathrm{l}$ of $5 \mu \mathrm{M}$ forward and reverse primer, and $1 \mu \mathrm{l}$ cDNA] was directly monitored by the Bioer FQD-66A detection system. The primers used were: rat SOD, forward, 5'-CCACTGCAGGACCTCATTTT-3' and reverse, 5'-CACCTTTGCCCAAGTCATCT-3'; rat GSH-PX, forward, 5'-GTCCACCGTGTATGCCTTCT-3' and reverse, 5'-CATT CACCTCGCACTTCTCA-3'; GAPDH, forward, 5'-ATTGT CAGCAATGCATCCTGCA-3' and reverse, 5'-AGACA ACCTGGTCCTCAGTGTA-3'. After an initial denaturation step at $95^{\circ} \mathrm{C}$ for $15 \mathrm{~min}$, temperature cycling was initiated. Each cycle consisted of denaturation at $94^{\circ} \mathrm{C}$ for $3 \mathrm{~min}$, annealing at $56^{\circ} \mathrm{C}$ for $30 \mathrm{sec}$, and elongation at $72^{\circ} \mathrm{C}$ for $30 \mathrm{sec}$. A total of 40 cycles were performed. We used GAPDH to normalize mRNA. Relative quantification of mRNA expression levels was determined using the relative standard curve method according to the manufacturer's instructions.

Activity of SOD and GSH-PX was assessed by spectrophotometry analysis using commercial kits: superoxide dismutase enzymatic activity assay kit, cat. no. A001-1 (Jiancheng Biotech, Nanjing, China); GSH-PX enzymatic activity assay kit, cat. no. A005 (Jiancheng Biotech). These two kits detected the remaining substrate quantity following SOD or GSH-PX enzymatic activity. An increase in optical density indicates a reduction of enzymatic activity. Optical density values were measured at emission wavelengths of $550 \mathrm{~nm}$ for SOD or $412 \mathrm{~nm}$ for GSH-PX. 
A

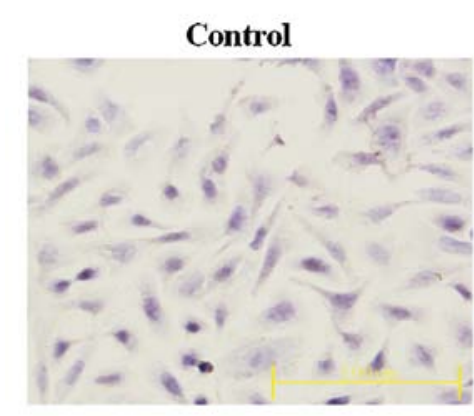

With AGEs $(400 \mu \mathrm{g} / \mathrm{ml})$

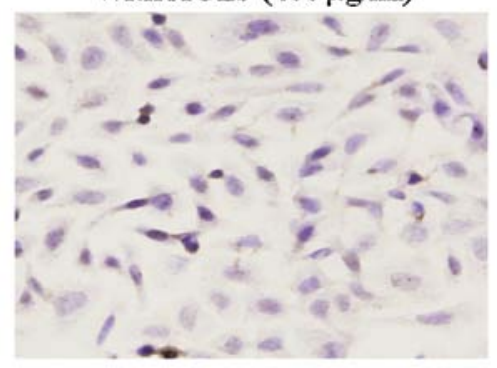

B

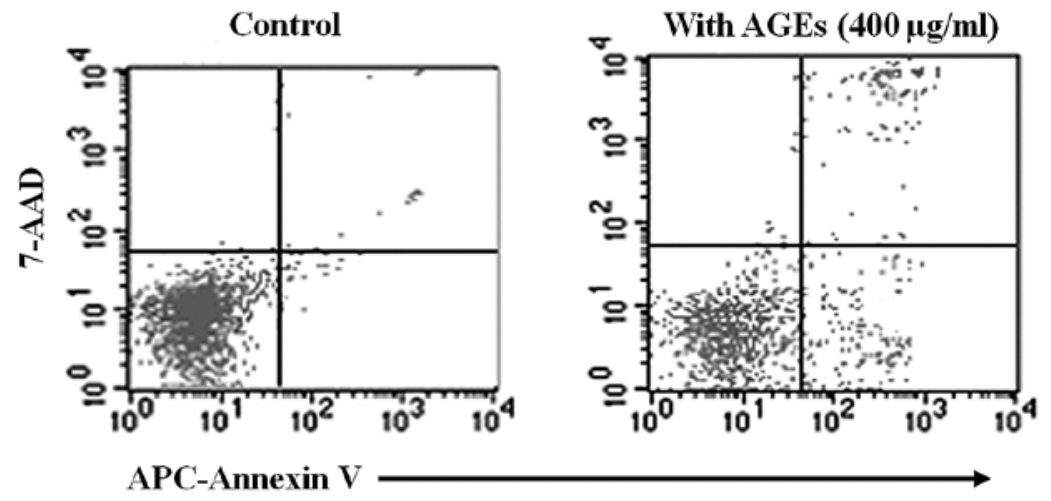

C

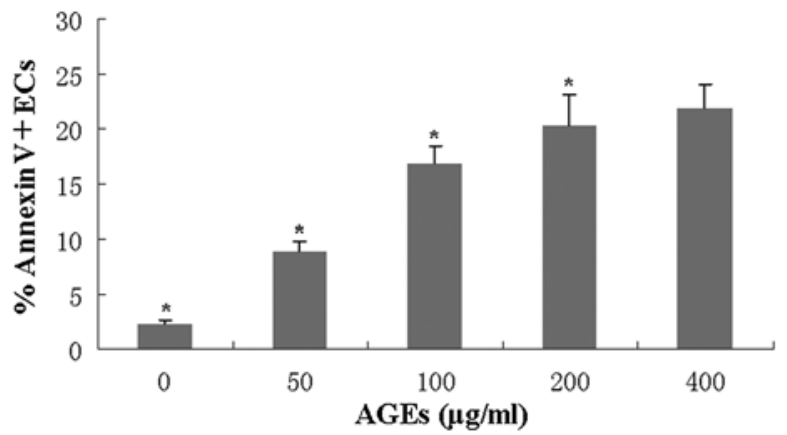

Figure 1. Effects of AGEs on EC apoptosis. ECs were co-cultured with AGEs $(0,50,100,200$ and $400 \mu \mathrm{g} / \mathrm{ml})$ for $24 \mathrm{~h}$. (A) Representative images of TUNEL staining. The nuclei of apoptotic cells appear brown. (B) Representative images of apoptosis, as analyzed by FACS. (C) Quantification of the Annexin V-positive ECs. " $\mathrm{P}<0.05$ group comparison. Data are presented as the means \pm SEM $(n=6)$.

Western blot analysis. The expressions of Bcl-2 and Bax were analyzed by western blotting. Briefly, total cell lysate was separated by SDS-PAGE (10\% resolving gel) and transferred to a polyvinylidine fluoride (PVDF) membrane (Roche, Basel, Switzerland) by electroblotting for $2 \mathrm{~h}$ at $100 \mathrm{~mA}$. The membrane was immunoblotted with antibodies against $\mathrm{Bcl}-2$ or Bax (both from Santa Cruz Biotechnology, Inc.) at $4^{\circ} \mathrm{C}$ overnight. Immunoreactivity was detected using the enhanced chemiluminescence reaction system (Amersham Pharmacia Biotech, Piscataway, NJ, USA) according to the manufacturer's instructions. GAPDH was used as a loading control. The expression of each protein was quantified by scanning densitometry and normalized against GAPDH. Data were expressed as a relative optical density value.

Statistical analysis. Data are presented as the means \pm SEM. SPSS v13.0 software (SPSS, Inc., Chicago, IL, USA) was used for statistical analysis. Differences among groups were evaluated by the unpaired Student's t-test or one-way ANOVA followed by a post hoc test. Values of $\mathrm{P}<0.05$ were considered to indicate statistically significant differences.

\section{Results}

HRM protects AGE-induced endothelial apoptosis. To detect the EC apoptosis induced by the presence of AGEs, cultured ECs were treated with AGEs and cultured in the normal or HRM-containing media prior to TUNEL staining. TUNEL staining showed that AGEs $(400 \mu \mathrm{g} / \mathrm{ml})$ induced apoptosis of ECs (Fig. 1A, right panel). In addition, flow cytometry analysis revealed that AGEs induced EC apoptosis in a concentrationdependent manner (Fig. 1B and C). After $24 \mathrm{~h}$, evaluation of the 4 EC groups, including the AGEs (400 $\mu \mathrm{g} / \mathrm{ml})$ group, HRM group, HRM + AGEs group, and normal control group, revealed that the cell apoptosis rate detected by flow cytometry was not significantly different between the HRM group and the 
A
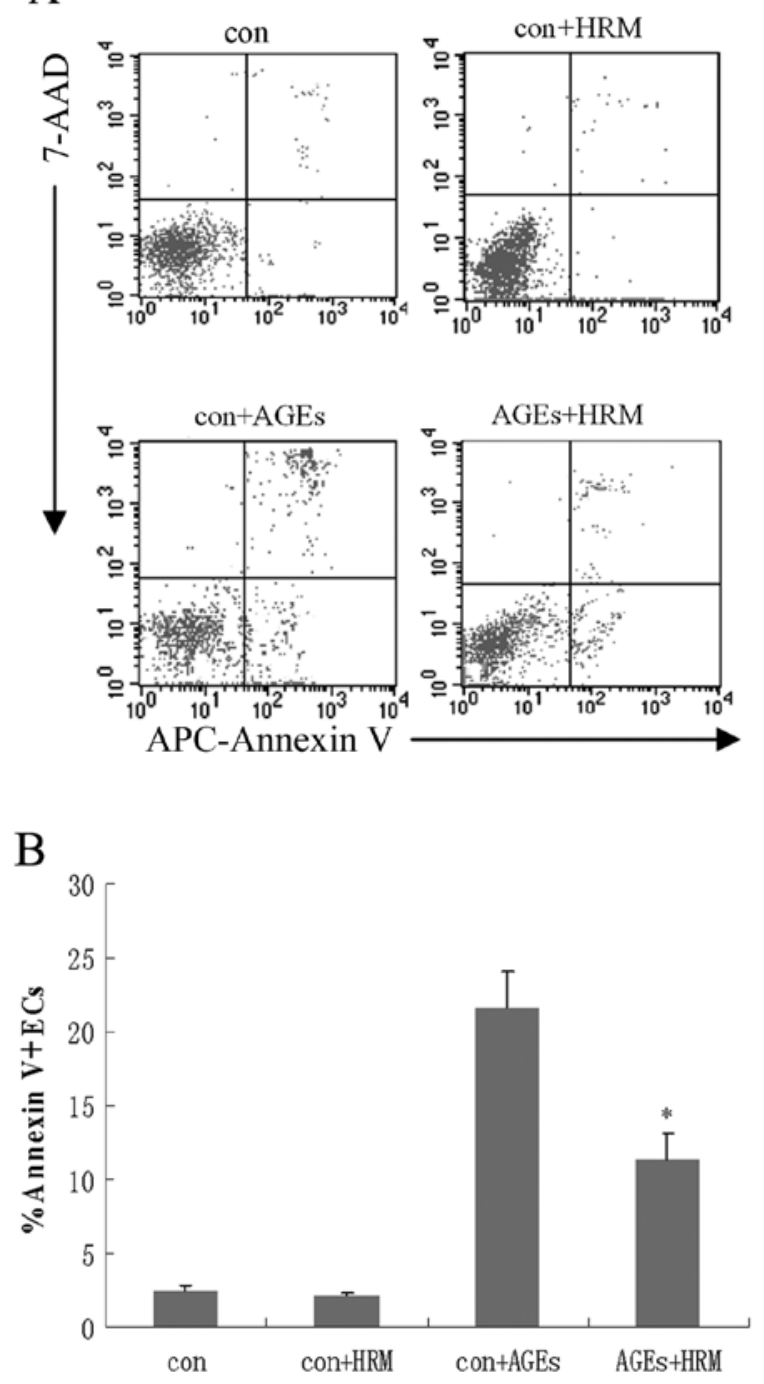

Figure 2. Effects of hydrogen-rich medium (HRM) on AGE-induced EC apoptosis. The ECs were cultured in the presence or absence of AGEs and in normal media or HRM for $24 \mathrm{~h}$ prior to Annexin V/7-AAD staining. (A) The representative results of APC-Annexin V/7-AAD staining and analysis by flow cytometry. (B) Quantification of the Annexin V-positive ECs. "P<0.05 vs. con + AGEs group. Data are presented as the means \pm SEM $(n=6)$.

control group when AGEs were absent. However, the presence of AGEs increased the rate of EC apoptosis, and the addition of HRM reduced the apoptosis rate of AGE-treated ECs from $21.61 \pm 2.52$ to $11.32 \pm 1.75 \%$ (Fig. 2).

$H R M$ reduces AGE-induced ROS generation in ECs. The production of intracellular ROS was measured by detection of DCF-DA by flow cytometry analysis. Treatment of ECs with AGEs significantly increased the generation of intracellular ROS in a dose-dependent manner (Fig. 3A). However, HRM significantly decreased AGE-induced intracellular ROS generation (Fig. 3B).

Effects of HRM on antioxidative enzymes in ECs exposed to AGEs. Co-culture with HRM and AGEs significantly reduced the level of SOD mRNA, and 50, 100, 200 and $400 \mu \mathrm{g} / \mathrm{ml}$ AGEs decreased the endothelial SOD mRNA levels to 76.34, 67.52, 40.03 and $39.15 \%$ of the baseline levels, respectively. However,
A
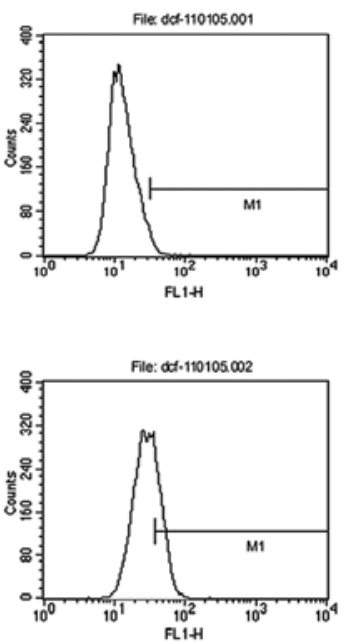

B

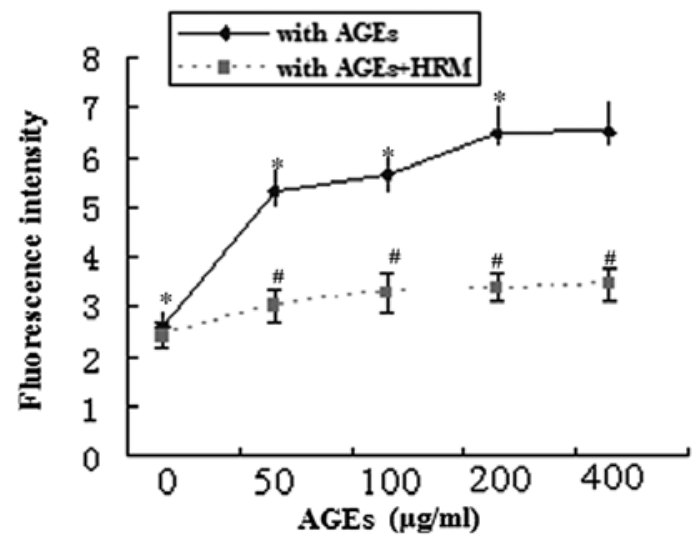

Figure 3. Effects of hydrogen-rich medium (HRM) on AGE-induced ROS production in ECs. ECs were cultured in the normal media or HRM with the addition of various concentrations of AGEs $(0,50,100,200$ and $400 \mu \mathrm{g} / \mathrm{ml})$ for $24 \mathrm{~h}$. Following cell culture, ECs were harvested and used in the ROS detection using flow cytometry after incubating with $10 \mathrm{mM}$ DCF-DA for $1 \mathrm{~h}$. (A) The representative results of flow cytometry to detect ROS using DCF-DA. Top panel, ECs in normal medium stained with DCF-DA; lower panel, ECs cultured in normal medium with Rosup and stained with DCFDA. (B) ECs cultured either in normal medium or HRM with the addition of various concentrations of AGEs were stained with DCF-DA and fluorescence quantified using flow cytometry. ${ }^{*} \mathrm{P}<0.05$ group comparison; \# $\mathrm{P}<0.05$ vs. the AGEs group. Data are presented as the means \pm SEM $(n=6)$.

following HRM intervention, the SOD expression reduction caused by AGEs was significantly attenuated (Fig. 4A). In addition, the enzyme activities of SOD and GSH-PX were determined by enzymatic activity assay, in which the optical density increases as the enzyme activity levels decrease. AGE treatment significantly increased the optical density of antioxidative enzymes. HRM treatment was also able to partly obstruct this effect (Fig. 4C and D).

Effects of HRM on the ratio of Bcl-2/Bax in ECs exposed to AGEs. Examination of the anti-apoptotic protein Bcl-2 and the pro-apoptotic protein Bax in ECs revealed that ECs co-cultured with AGEs showed decreased Bcl-2 protein levels 

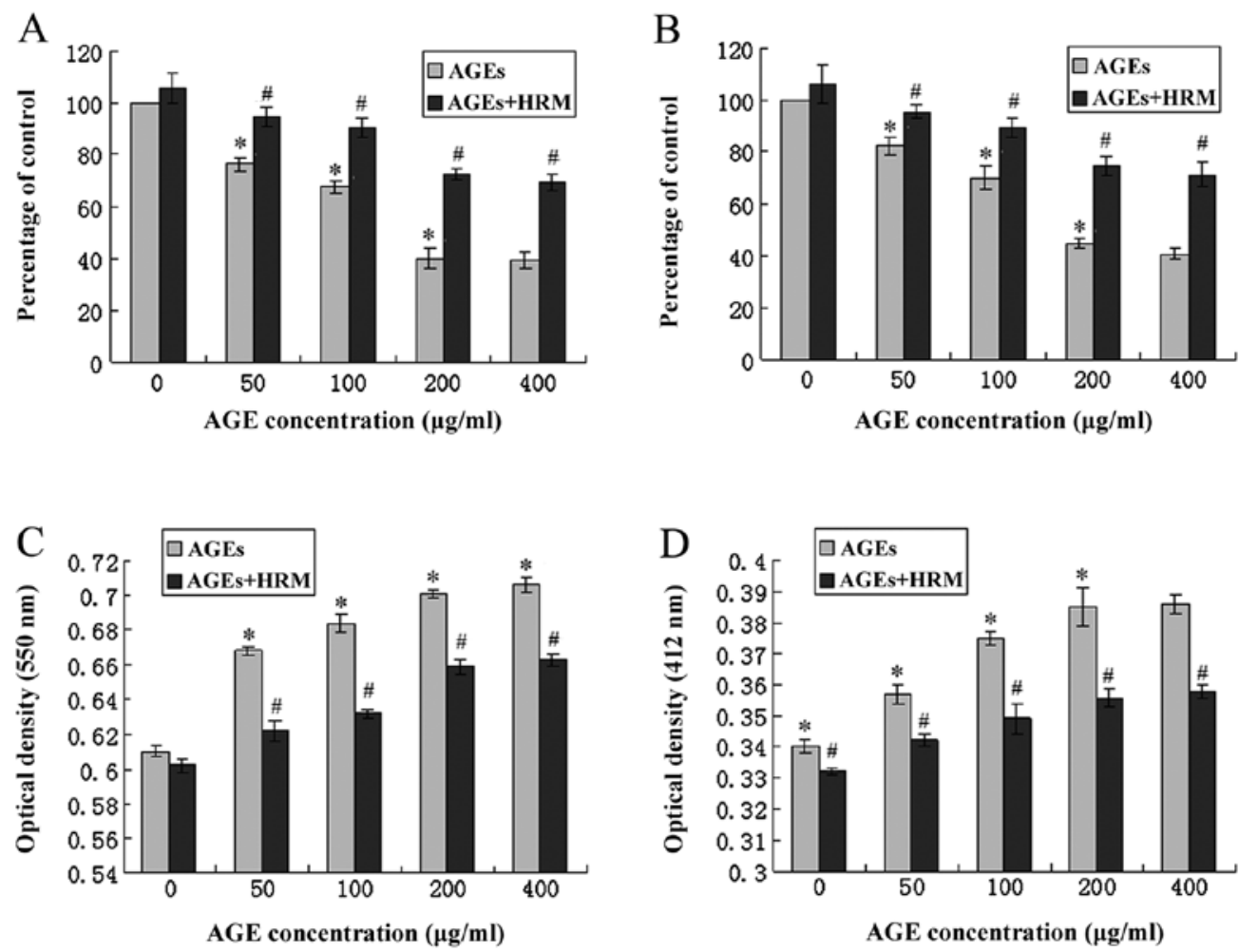

Figure 4. Effects of hydrogen-rich medium (HRM) on antioxidative enzyme expression. Detection of antioxidative gene expression in ECs cultured in the normal medium or HRM with the addition of various concentrations of AGEs. (A) Real-time PCR detection of SOD mRNA expression relative to GAPDH mRNA. (B) Real-time PCR detection of GSH-PX mRNA expression relative to GAPDH mRNA. (C) The detection of SOD protein level by enzymatic activity assay. (D) The detection of GSH-PX protein expression by enzymatic activity assay. ${ }^{*} \mathrm{P}<0.05$ group comparison; ${ }^{*} \mathrm{P}<0.05$ vs. the AGEs group. Data are presented as the means \pm SEM $(n=6)$.

A

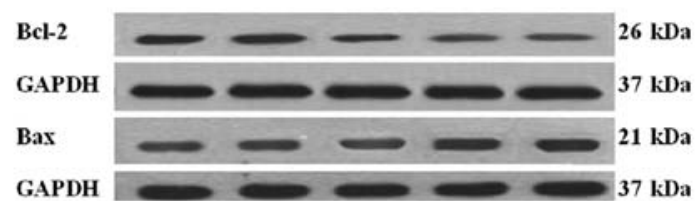

B

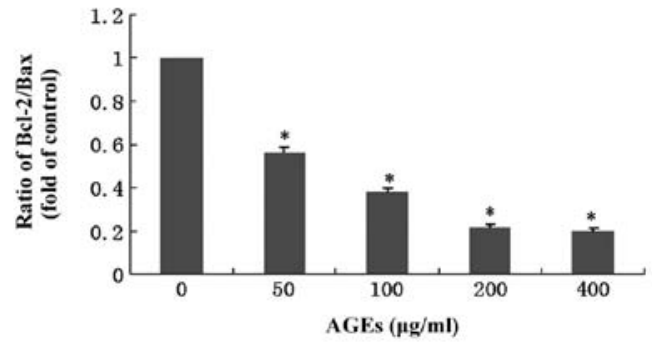

C

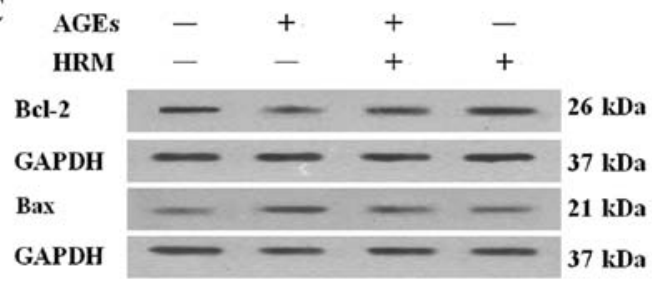

D

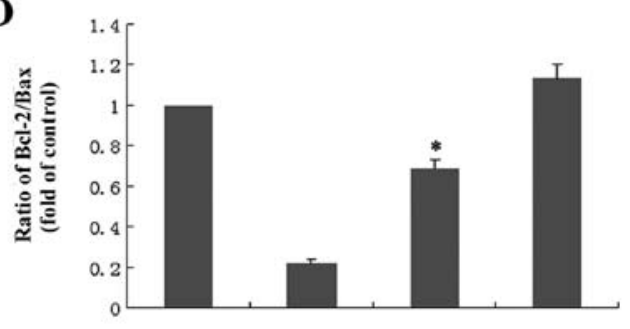

Figure 5. Effects of hydrogen-rich medium (HRM) on the ratio of Bcl-2/Bax in ECs exposed to AGEs. Bcl-2 and Bax protein expression levels were normalized against the expression levels of the loading control, GAPDH. The results are expressed as the means \pm SEM ( $\mathrm{n}=6$ ). (A) The Bcl-2, Bax proteins in ECs cultured in $0,50,100,200$ and $400 \mu \mathrm{g} / \mathrm{ml}$ of AGEs were detected by western blot analysis. (B) Quantification of Bcl-2/Bax protein expression ratio from ECs cultured in 0,50, 100, 200 and $400 \mu \mathrm{g} / \mathrm{ml}$ of AGEs. "P $<0.05$ vs. control. (C) The Bcl-2, Bax proteins in ECs cultured in normal media or HRM in the presence of $400 \mu \mathrm{g} / \mathrm{ml}$ AGEs were detected by western blot analysis. (D) Quantification of Bcl-2/Bax protein expression ratio from ECs cultured in normal media or HRM in the presence of $400 \mu \mathrm{g} / \mathrm{ml}$ AGEs. ${ }^{*} \mathrm{P}<0.05$ vs. the AGEs group.

and increased Bax protein expression. The ratio of $\mathrm{Bcl}-2 / \mathrm{Bax}$ was decreased by $\sim 50 \%$ following exposure to $400 \mu \mathrm{g} / \mathrm{ml}$ AGEs (Fig. 5A and B), indicating that EC apoptosis induced by AGEs occurs partly through the mitochondrial apoptotic pathway. HRM was shown to attenuate AGE-induced decrease in the ratio of Bcl-2/Bax (Fig. 5C and D). 


\section{Discussion}

To the best of our knowledge, this study is the first to report that HRM reduces AGE-induced apoptosis in ECs, and this reduction in $\mathrm{EC}$ apoptosis was associated with the reduction of ROS and increase of the Bcl-2/Bax ratio. Evidence from the present study as well as from our previous studies $(6,22)$ support this conclusion. Although HRM has no significant protective effects against endothelial apoptosis under normal culture conditions, it can significantly reduce EC apoptosis induced by large doses of AGEs. AGEs increase intracellular ROS in a concentration-dependent manner, and HRM is able to reduce ROS increases induced by different concentrations of AGEs $(6,22)$. AGEs also reduce expression of SOD and GSH-PX in ECs, which is important as these enzymes have been shown to play a role in antagonizing ROS $(23,24)$. SOD and GSH-PX activity is reduced by AGEs in a concentrationdependent manner, and HRM intervention is shown to partially antagonize AGE-induced reduction of antioxidant enzyme expression and activity. Additionally, AGEs can reduce the expression of anti-apoptotic protein $\mathrm{Bcl}-2$ and increase the expression of pro-apoptotic protein Bax, thereby reducing the $\mathrm{Bcl}-2 / \mathrm{Bax}$ ratio. HRM was also able to ameliorate this activity, which partially reverses the AGE-induced reduction in the Bcl-2/Bax ratio.

A high glucose environment can lead to excessive production of AGEs in the body. It has been reported that AGEs can significantly increase the ROS content in ECs and promote apoptosis, and EC injury is closely associated with atherosclerosis (4). Results of our previous study showed that the receptor for AGEs (RAGE) is key to the inflammatory process and endothelial activation, making it likely to accelerate atherosclerosis, particularly in diabetes patients (6). Results of that study also showed that high concentrations of C-reactive protein (CRP) may decrease the antioxidant defenses of endothelial progenitor cells (EPCs) by upregulating RAGE, promoting EPC sensitivity toward apoptosis mediated by oxidative stress (6). Oxidative stress and EC injury have been extensively investigated $(4,6,22,25)$, and ROS play an important role in AGE-induced cell injury (25). The RAGE activation mechanism was shown to decrease antioxidative enzyme activities, not only through increasing ROS production, but also by downregulating antioxidative enzyme mRNA expression (6). In the present study, we found that AGEs can lead to increased ROS production in rat ECs, resulting in apoptosis. Therefore, inhibiting the increase of AGE-caused ROS generation may be an effective method to mitigate EC injury.

Several studies have demonstrated the protective effects of $\mathrm{H}_{2}$ by using HRM to elevate cellular antioxidative defense mechanisms $(8,13,16,18,26-31)$. Yu et al (28) identified the protective effects of HRM on human epidermal fibroblasts under oxidative stress caused by diabetes. The investigators suggested that $\mathrm{H}_{2}$ was a potential protective antioxidant for both preventive and therapeutic applications through its reduction of the hydroxyl radical, which is the most cytotoxic of ROS (8). Similar protective effects were demonstrated in liver injury models as $\mathrm{H}_{2}$ selectively reduced the strongest oxidants $(\cdot \mathrm{OH}$ and $\mathrm{ONOO}^{-}$) without interfering with metabolic oxidation-reduction reactions or ROS cell signaling (16). Hydrogen-rich PBS exhibited protective effects against radiation-induced cellular damage, suggesting that $\mathrm{H}_{2}$ may be an effective radioprotective agent (27). $\mathrm{H}_{2}$ also has protective effects against myocardial ischemia and reperfusion (30). Additionally, hydrogen-rich saline protected the myocardium against ischemia/reperfusion in a rat model, resulting in improved left ventrical systolic and diastolic pressure (13). Chronic hydrogen-rich saline treatment was also shown to reduce left ventricular hypertrophy caused by hypertension by ablating oxidative stress, suppressing inflammation and preserving mitochondrial function (29). $\mathrm{H}_{2}$ has also been shown to reduce the plasma glucose levels of diabetic patients (26), alleviate atherosclerosis in apolipoprotein E knockout mice (18), and provide protective effects against high-fat, diet-induced atherosclerosis (31). Moreover, to date, no significant side-effects or toxic effects of $\mathrm{H}_{2}$ have been found. $\mathrm{H}_{2}$ reacts with $\cdot \mathrm{OH}$, yielding water; $\mathrm{H}_{2}$ has a low molecular weight so that it easily enters intracellular structures where it plays an active role (32).

The present study adopted HRM to observe the protective effects of $\mathrm{H}_{2}$ on AGE-treated ECs. We showed that increasing $\mathrm{H}_{2}$ levels through the use of HRM alleviated AGE-induced oxidative injury of ECs. HRM attenuated ROS increases in ECs following AGE treatment, increased SOD and GSH-PX expression, reduced apoptosis, and simultaneously elevated the $\mathrm{Bcl}-2 / \mathrm{Bax}$ ratio. Briefly, our results showed that $\mathrm{H}_{2}$ can significantly reduce intracellular ROS levels and AGE-induced apoptosis, indicating that $\mathrm{H}_{2}$ may reduce AGE-induced cell apoptosis and protect ECs through mitigating cellular oxidative stress. This is in accordance with the results of a previous report, which showed that AGEs can reduce the generation of antioxidant enzymes in the cells, and destroy the balance of cellular oxidation within the antioxidant system, which affects endothelial function (33). The expression of antioxidant enzymes, SOD and GSH-PX, protects against glucose-induced oxidative stress in vascular contractile cells and AGE-induced endothelial injury (34). As an essential active reductase in cell metabolism, the enzyme SOD plays an important role in preventing organisms from oxidative damage, and GSH-PX clears lipid peroxides induced by $\mathrm{ROS}$ and $\cdot \mathrm{OH}$, protecting the integrity of cell membrane structure and function (28). Liu et al (12) also showed that hydrogen-rich saline markedly increased the activities of antioxidant enzymes SOD and GSH in a rat model of liver damage. In the present study, HRM significantly reduced the degree to which SOD and GSH-PX were inactivated in cells treated with AGEs. We found that the application of $\mathrm{H}_{2}$ significantly precluded AGE-induced decline in the levels of those two enzymes in ECs, indicating that $\mathrm{H}_{2}$ protects the EC antioxidant system from AGE-induced injury and stabilizes cell function.

AGE-induced apoptosis in ECs has been shown to be related to decreased $\mathrm{Bcl}-2 / \mathrm{Bax}$ ratio (35). The $\mathrm{Bcl}-2$ protein exerts anti-apoptotic effects through antioxidant activity or inhibition of ROS generation (36). By contrast, the Bax gene plays an inductive role, promoting apoptosis through antagonizing the Bcl-2 gene (23). A previous study by Oltvai et al (24) suggested that the ratio of Bcl-2 to Bax determines apoptosis or inhibition of apoptosis. In the present study, we examined the anti-apoptotic protein Bcl-2 and the pro-apoptotic protein Bax in ECs, finding that ECs co-cultured with AGEs had decreased $\mathrm{Bcl}-2$ protein levels and increased Bax protein expression. The ECs cultured with AGEs in HRM had significantly decreased 
apoptosis levels, while the $\mathrm{Bcl}-2 / \mathrm{Bax}$ ratio was elevated. We suggest that it is possible that the $\mathrm{H}_{2}$ protective mechanism is to upregulate $\mathrm{Bcl}-2 / \mathrm{Bax}$, thereby reducing $\mathrm{EC}$ apoptosis induced by AGEs and protecting EC function.

In conclusion, $\mathrm{H}_{2}$ has significant protective effects against AGE-caused EC injury. The mechanism of its protective effects may be to reduce ROS generation, protect the intracellular antioxidant enzyme system, and elevate the Bcl-2/Bax ratio. Further studies are required to explore the specific mechanism of the protective effects of $\mathrm{H}_{2}$ against AGE-induced EC injury.

\section{Acknowledgements}

This study was supported in part by the National Natural Science Foundation of China (Grants 30872710, 30770852).

\section{References}

1. Faria AM, Papadimitriou A, Silva KC, Lopes de Faria JM and Lopes de Faria JB: Uncoupling endothelial nitric oxide synthase is ameliorated by green tea in experimental diabetes by re-establishing tetrahydrobiopterin levels. Diabetes 61: 18381847,2012

2. Caporali A, Pani E, Horrevoets AJ, et al: Neurotrophin p75 receptor (p75NTR) promotes endothelial cell apoptosis and inhibits angiogenesis: implications for diabetes-induced impaired neovascularization in ischemic limb muscles. Circ Res 103: e15-e26, 2008.

3. Csiszar A and Ungvari Z: Endothelial dysfunction and vascular inflammation in type 2 diabetes: interaction of AGE/RAGE and TNF-alpha signaling. Am J Physiol Heart Circ Physiol 295: H475-H476, 2008.

4. Goldin A, Beckman JA, Schmidt AM and Creager MA: Advanced glycation end products: sparking the development of diabetic vascular injury. Circulation 114: 597-605, 2006.

5. Xiang M, Yang M, Zhou C, Liu J, Li W and Qian Z: Crocetin prevents AGEs-induced vascular endothelial cell apoptosis Pharmacol Res 54: 268-274, 2006.

6. Chen J, Huang L, Song M, Yu S, Gao P and Jing J: C-reactive protein upregulates receptor for advanced glycation end products expression and alters antioxidant defenses in rat endothelial progenitor cells. J Cardiovasc Pharmacol 53: 359-367, 2009.

7. Tan AL, Forbes JM and Cooper ME: AGE, RAGE, and ROS in diabetic nephropathy. Semin Nephrol 27: 130-143, 2007.

8. Ohsawa I, Ishikawa M, Takahashi $\mathrm{K}$, et al: Hydrogen acts as a therapeutic antioxidant by selectively reducing cytotoxic oxygen radicals. Nat Med 13: 688-694, 2007.

9. Xie K, Yu Y, Huang Y, et al: Molecular hydrogen ameliorates lipopolysaccharide-induced acute lung injury in mice through reducing inflammation and apoptosis. Shock 37: 548-555, 2012.

10. Cai J, Kang Z, Liu K, et al: Neuroprotective effects of hydrogen saline in neonatal hypoxia-ischemia rat model. Brain Res 1256: 129-137, 2009.

11. Chen H, Sun YP, Li Y, et al: Hydrogen-rich saline ameliorates the severity of 1-arginine-induced acute pancreatitis in rats. Biochem Biophys Res Commun 393: 308-313, 2010.

12. Liu Q, Shen WF, Sun HY, et al: Hydrogen-rich saline protects against liver injury in rats with obstructive jaundice. Liver Int 30: 958-968, 2010

13. Sun Q, Kang Z, Cai J, et al: Hydrogen-rich saline protects myocardium against ischemia/reperfusion injury in rats. Exp Biol Med 234: 1212-1219, 2009.

14. Li J, Wang C, Zhang JH, Cai JM, Cao YP and Sun XJ: Hydrogen-rich saline improves memory function in a rat model of amyloid-beta-induced Alzheimer's disease by reduction of oxidative stress. Brain Res 1328: 152-161, 2010.

15. Sun Q, Cai J, Zhou J, Tao H, Zhang JH, Zhang W and Sun XJ: Hydrogen-rich saline reduces delayed neurologic sequelae in experimental carbon monoxide toxicity. Crit Care Med 39: 765-769, 2011.
16. Sun $\mathrm{H}$, Chen L, Zhou W, et al: The protective role of hydrogenrich saline in experimental liver injury in mice. J Hepatol 54: 471-480, 2011.

17. Terasaki Y, Ohsawa I, Terasaki M, et al: Hydrogen therapy attenuates irradiation-induced lung damage by reducing oxidative stress. Am J Physiol Lung Cell Mol Physiol 301: L415-L426, 2011.

18. Ohsawa I, Nishimaki K, Yamagata K, Ishikawa M and Ohta S: Consumption of hydrogen water prevents atherosclerosis in apolipoprotein E knockout mice. Biochem Biophys Res Commun 377: 1195-1198, 2008.

19. Qin ZX, Yu P, Qian DH, et al: Hydrogen-rich saline prevents neointima formation after carotid balloon injury by suppressing ROS and the TNF- $\alpha / \mathrm{NF}-\kappa \mathrm{B}$ pathway. Atherosclerosis 220 : 343-350, 2012.

20. Wu X, Zhou Q, Huang L, Sun A, Wang K, Zou Y and Ge J: Ageing-exaggerated proliferation of vascular smooth muscle cells is related to attenuation of Jagged1 expression in endothelial cells. Cardiovasc Res 77: 800-808, 2008.

21. Tang X, Guo Y, Nakamura K, et al: Nitroalkenes induce rat aortic smooth muscle cell apoptosis via activation of caspase-dependent pathways. Biochem Biophys Res Commun 397: 239-244, 2010.

22. Chen J, Song M, Yu S, Gao P, Yu Y, Wang H and Huang L: Advanced glycation endproducts alter functions and promote apoptosis in endothelial progenitor cells through receptor for advanced glycation endproducts mediate overpression of cell oxidant stress. Mol Cell Biochem 335: 137-146, 2010.

23. Marsh SA, Laursen PB, Pat BK, Gobe GC and Coombes JS $\mathrm{Bcl}-2$ in endothelial cells is increased by vitamin $\mathrm{E}$ and alphalipoic acid supplementation but not exercise training. J Mol Cell Cardiol 38: 445-451, 2005.

24. Oltvai ZN, Milliman CL and Korsmeyer SJ: Bcl-2 heterodimerizes in vivo with a conserved homolog, Bax, that accelerates programmed cell death. Cell 74: 609-619, 1993.

25. Jandeleit-Dahm K, Watson A and Soro-Paavonen A: The AGE/ RAGE axis in diabetes-accelerated atherosclerosis. Clin Exp Pharmacol Physiol 35: 329-334, 2008.

26. Kajiyama S, Hasegawa G, Asano M, et al: Supplementation of hydrogen-rich water improves lipid and glucose metabolism in patients with type 2 diabetes or impaired glucose tolerance. Nutr Res 28: 137-143, 2008.

27. Qian L, Li B, Cao F, Huang Y, Liu S, Cai J and Gao F: Hydrogen-rich PBS protects cultured human cells from ionizing radiation-induced cellular damage. Nucl Technol Radiat Prot 25: 23-29, 2010.

28. Yu P, Wang Z, Sun X, Chen X, Zeng S, Chen L and Li S: Hydrogen-rich medium protects human skin fibroblasts from high glucose or mannitol induced oxidative damage. Biochem Biophys Res Commun 409: 350-355, 2011.

29. Yu Ys and Zheng H: Chronic hydrogen-rich saline treatment reduces oxidative stress and attenuates left ventricular hypertrophy in spontaneous hypertensive rats. Mol Cell Biochem 365: 233-242, 2012.

30. Zhang Y, Sun Q, He B, Xiao J, Wang Z and Sun X: Antiinflammatory effect of hydrogen-rich saline in a rat model of regional myocardial ischemia and reperfusion. Int J Cardiol 148: 91-95, 2011.

31. Zong C, Song G, Yao S, et al:Administration of hydrogensaturated saline decreases plasma low-density lipoprotein cholesterol levels and improves high-density lipoprotein function in high-fat diet-fed hamsters. Metabolism 61: 794-800, 2012.

32. Schoenfeld MP, Ansari RR, Zakrajsek JF, et al: Hydrogen therapy may reduce the risks related to radiation-induced oxidative stress in space flight. Med Hypotheses 76: 117-118, 2011.

33. Hess DA and Hegele RA: Linking diabetes with oxidative stress, adipokines, and impaired endothelial precursor cell dunction. Can J Cardiol 28: 629-630, 2012.

34. Sharpe PC, Liu WH, Yue KK, et al: Glucose-induced oxidative stress in vascular contractile cells: comparison of aortic smooth muscle cells and retinal pericytes. Diabetes 47: 801-809, 1998.

35. Li BY, Li XL, Cai Q, et al: Induction of lactadherin mediates the apoptosis of endothelial cells in response to advanced glycation end products and protective effects of grape seed procyanidin B2 and resveratrol. Apoptosis 16: 732-745, 2011.

36. Deng X, Gao F and May WS Jr: Bcl2 retards G1/S cell cycle transition by regulating intracellular ROS. Blood 102: 3179 $3185,2003$. 